\title{
A Patient With Psychotic Disorder Following Traumatic Brain Injury With Bilateral Frontal Lobe Damage
}

\author{
Halil Onder ${ }^{\mathrm{a}, \mathrm{c}}$, Selim Sagir ${ }^{\mathrm{b}}$
}

\section{To the Editor}

We evaluated a 43-year-old male patient in his routine policlinic controls due to chronic psycosis and impulsive behavior disorder. Upon history taking, it was learnt that the patient had been hospitalized due to subdural and bilateral frontal hemorrhage following a car accident 9 years ago. After discharge, the patient was unable to work due to prominent behavioral changes and cognitive problems. Aggressive and impulsive behavior changes were apparent. In addition, it was learnt that the patient demostrated hypersexuality in the form of inappropriate remarks and attempts to touch his wife in public place. Besides, he expressed frequently auditory hallucinations characterized by hearing recurrent wind rustles and persecutory delusions. There was not a past psychiatric disorder, drug or alcohol usage or regular medications prior to the accident and not a family history of any psychiatric disorder was taken. During psychological evaluations, his mood was apathic, but his affect could change suddenly aggressively particularly towards his wife. Of note, the patient's symptoms had started soon after the accident and not a progression had occurred over the years. On neurological examination, there was a partial failure in cooperation and orientation examinations which was associated with moderate cognitive impairment and apathy. Other examinations including motor, sensorial and cerebellar tests were in normal ranges. Mini-mental state examination score was $12 / 30$, which was prominently disturbed in attentional abilities, but preserved short-term memory (3/3). Cranial magnetic resonance imaging (MRI) showed bilateral frontal encephalomalasia associated with previous traumatic brain injury (Fig. 1). Taken together the clinical and neuroimaging findings, we established the diagnosis of psychotic disorder due to traumatic brain injury. Dosage increment in the antipsychotic medication was made (risperidone from 1 to $1.5 \mathrm{mg}$ once a day). Follow-up in the outpatient clinic was planned.

\section{Discussion}

Here, we present a patient with psychotic disorder following

Manuscript accepted for publication March 30, 2017

${ }^{a}$ Department of Neurology, Yozgat State Hospital, Yozgat, Turkey

a'Department of Psychiatry, Yozgat State Hospital, Yozgat, Turkey

${ }^{c}$ Corresponding Author: Halil Onder, Department of Neurology, Yozgat State

Hospital, Yozgat, Turkey. Email: halilnder@yahoo.com

doi: https://doi.org/10.14740/jnr423w traumatic brain injury which was located in bifrontal regions. Considering the accurate temporal relationship between the brain trauma and following psychotic symptoms, we think that this case represents a smart sample of a patient with psychotic symptoms which were associated with traumatic brain injury. Following the accident, the patient had suffered a moderate cognitive impairment, and apparent behavioral changes including apathy and impulsivity. A major discussion may be that posttraumatic neurocognitive disturbances may mimic disorganized speech, the disturbances of thought and behavior which can be mistakenly interpreted as psychotic disorder [1]. However, in our patient, auditory hallucinations and persecutory delusions were prominent which enabled the diagnosis of psychotic disorder. Accordingly, persecutory delusions are reported to be in the forefront of the clinical presentations in patients with posttraumatic psychosis [1]. The cranial MRI of our patient showed bilateral frontal severe encephalomacia. It has also been reported that rate of neuroimaging abnormalities was considerably high $(65 \%)$ in post-traumatic psychosis [2] and frontal-temporal lobes were the most common areas affected in this patient group [1]. Another clue for the diagnosis of post-traumatic psychosis is accompanying cognitive impairment [2] which was prominent in executive functions in our patient. Nonetheless, the presence of other clinical signs of disturbed executive functions and emotional and motivational alterations might rather be attributed to frontal-subcortical system dysfunction and related syndromes (dorsolateral syndrome, orbitofrontal syndrome, and anterior cingulate syndrome), other than solely the process of psychosis. Hence, evaluating this patient as frontal lobe syndrome and accompanyging pscyhotic disorder may be more rational. In conclusion, this report illustrates an interesting patient with posttraumatic psychosis in addition to other frontal disturbances which constitutes a demonstrative report for clinical concerns. Future reports of larger cases of these patients in association with brain lesions will add substantial insights for understanding the anatomical pathways underlying psychosis as well as other neurological disorders. These study results may also add new perspectives to the unknown aspects of the pathophysiology of primary psychosis, from a distinct point of view.

\section{Conflicts of Interest}

None.

\section{References}

1. Arciniegas DB, Harris SN, Brousseau KM. Psychosis 


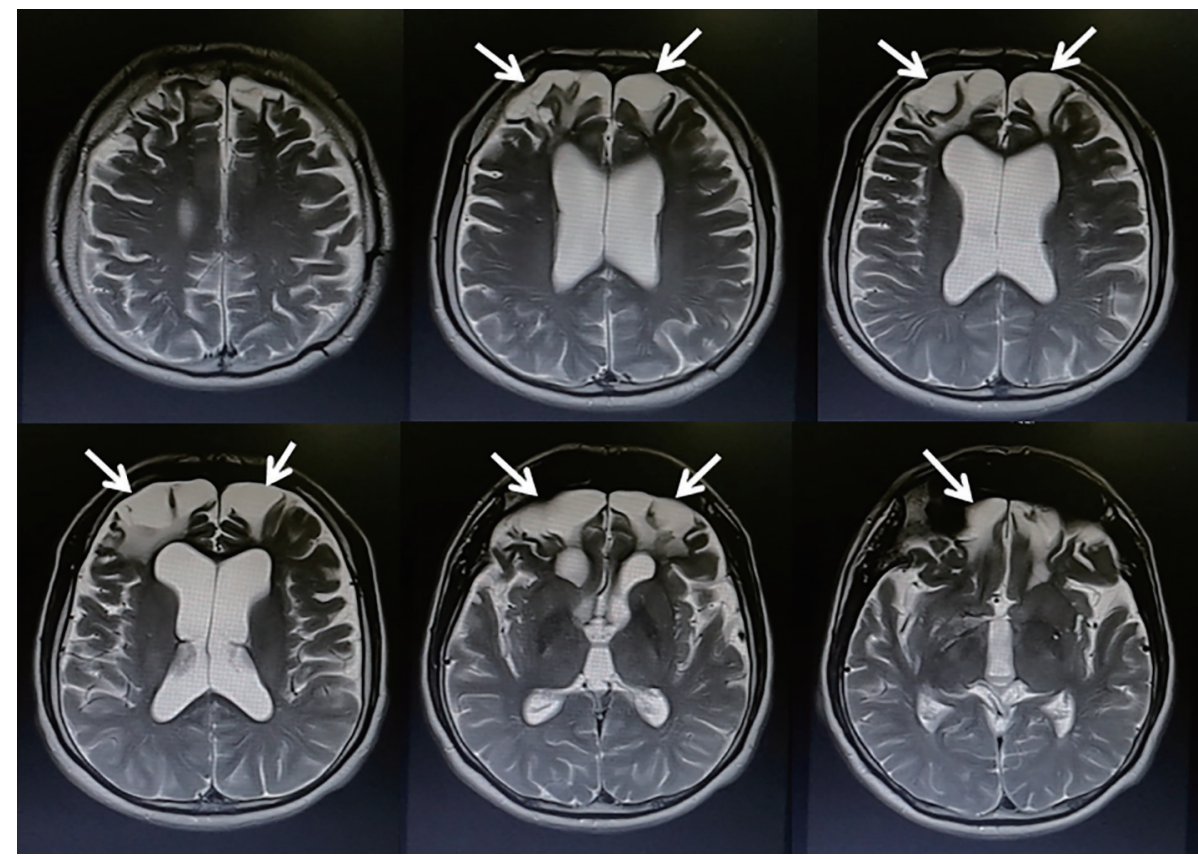

Figure 1. Cranial MRI showing encephalomalacia in the bilateral frontal lobes (arrows).

following traumatic brain injury. Int Rev Psychiatry. 2003;15(4):328-340.

2. Fujii D, Ahmed I. Characteristics of psychotic disorder due to traumatic brain injury: an analysis of case studies in the literature. J Neuropsychiatry Clin Neurosci. 2002;14(2):130-140. 